\title{
GENERIC FORMAL FIBERS AND ANALYTICALLY RAMIFIED STABLE RINGS
}

\author{
BRUCE OLBERDING
}

\begin{abstract}
Let $A$ be a local Noetherian domain of Krull dimension $d$. Heinzer, Rotthaus, and Sally have shown that if the generic formal fiber of $A$ has dimension $d-1$, then $A$ is birationally dominated by a 1-dimensional analytically ramified local Noetherian ring having residue field finite over the residue field of $A$. We explore further this correspondence between prime ideals in the generic formal fiber and 1-dimensional analytically ramified local rings. Our main focus is on the case where the analytically ramified local rings are stable, and we show that in this case the embedding dimension of the stable ring reflects the embedding dimension of a prime ideal maximal in the generic formal fiber, thus providing a measure of how far the generic formal fiber deviates from regularity. A number of characterizations of analytically ramified local stable domains are also given.
\end{abstract}

\section{$\S 1$. Introduction}

An important technical fact regarding a finitely generated algebra $A$ over a field or the ring of integers is that if $A$ is reduced, then $A$ has finite normalization, meaning that the integral closure of $A$ in its total ring of quotients is a finitely generated $A$-module. Geometrically, this guarantees that the normalization of a variety or arithmetic scheme is given by a finite morphism. However, outside the geometric and arithmetic contexts, a local Noetherian domain, even of dimension 1, need not have finite normalization. Well-known examples due to Akizuki, Schmidt, and Nagata attest to this (see [1], [31], and [21, Example 3, p. 205]). These examples are constructed between a carefully chosen rank 1 discrete valuation ring (DVR) and its completion, and as such, the examples suggest a certain esoteric quality regarding the failure of a 1-dimensional local Noetherian domain to have finite normalization. However, an entirely different construction due to Heinzer, Rotthaus,

Received April 19, 2011. Revised February 7, 2012. Accepted July 25, 2012.

First published online April 29, 2013.

2010 Mathematics Subject Classification. Primary 13E05, 13B35, 13B22; Secondary $13 \mathrm{~F} 40$.

(C) 2013 by The Editorial Board of the Nagoya Mathematical Journal 
and Sally [8, Corollary 1.27] suggests a certain inevitability to such examples and shows that local Noetherian domains without finite normalization can be found birationally dominating any $d$-dimensional local Noetherian domain, $d>1$, having generic formal fiber of dimension $d-1$ (which is the case if $A$ is essentially of finite type over a field).

More precisely, let $A$ be a local Noetherian domain with maximal ideal $\mathfrak{m}$ and quotient field $F$, and let $\widehat{A}$ denote the completion of $A$ in the $\mathfrak{m}$-adic topology. The generic formal fiber of $A$ is the localization of $\widehat{A}$ at the multiplicatively closed subset consisting of the nonzero elements of $A$. Thus, the generic formal fiber of $A$ is the Noetherian $\operatorname{ring} \widehat{A}[F]$, and the prime ideals of $\widehat{A}[F]$ are those prime ideals extended from prime ideals $P$ of $\widehat{A}$ such that $P \cap A=0$. When $A$ has Krull dimension $d>0$, then the generic formal fiber of $A$ has Krull dimension less than $d$. Heinzer, Rotthaus, and Sally [8, Corollary 1.27] have shown that if the generic formal fiber is as large as possible in the sense that its Krull dimension is $d-1$, then there exists an analytically ramified local Noetherian domain $R$ birationally dominating $A$ and having residue field finite over the residue field of $A$. A theorem of Krull (see [10]) asserts that the property of being analytically ramified, meaning that the completion contains nonzero nilpotent elements, is equivalent for 1-dimensional local Noetherian domains to the failure to have finite normalization. The description of the rings in [8] is quite straightforward: given a prime ideal $P$ of $\widehat{A}$ of dimension 1 such that $P \cap A=0$, the $\operatorname{ring} F \cap(\widehat{A} / I)$ is a 1-dimensional analytically ramified local Noetherian ring for appropriate choices of $P$-primary ideal $I$. (This is restated more formally in Lemma 5.1 below.) Moreover, Matsumura [18, Theorem 2] has shown that such a prime ideal $P$ exists in $\widehat{A}$ whenever $A$ is essentially of finite type over a field, so the construction is relevant in natural circumstances.

In this article we explore further the connection between the generic formal fiber of $A$ and the nature of the analytically ramified 1-dimensional local rings which birationally dominate it. Specifically, we show that the embedding dimension of birationally dominating analytically ramified "stable" local rings reflects the regularity, or lack thereof, of the generic formal fiber. To phrase this more precisely, we introduce some terminology. Recall that if $A \subseteq R$ is an extension of quasi-local domains, then $R$ dominates $A$ if the maximal ideal of $A$ is a subset of the maximal ideal of $R$, and if $A$ and $R$ share the same quotient field, then $R$ birationally dominates $A$. When $R$ birationally dominates $A$, and $R / \mathfrak{m} R$ is a finite $A$-module, then we say that $R$ finitely dominates $A$; if also $R=A+\mathfrak{m} R$, then $R$ tightly 
dominates $A$. Thus, in our terminology, the theorem of Heinzer, Rotthaus, and Sally [8, Corollary 1.27] states that when the generic formal fiber of the $d$-dimensional local Noetherian domain $A$ has dimension $d-1$, then $A$ is finitely dominated by a 1-dimensional analytically ramified local Noetherian domain.

Our focus is on a specific class of 1-dimensional analytically ramified local Noetherian domains, those that are "stable" in the sense of Lipman [11] and Sally and Vasconcelos [30]. These rings, which we define in Section 2, are characterized in the local Noetherian case by the property that each ideal has a principal reduction of reduction number at most 1 ; that is, for each ideal $I$ of $R$, there exists $i \in I$ such that $I^{2}=i I$. As we discuss in Section 2, analytically ramified local Noetherian stable domains of embedding dimension greater than 2 were previously known to exist only in characteristic 2 and in a special setting. One of the main results of the present article is that when $A$ is an excellent local Noetherian domain of dimension $d>1$, then the dimension of the generic formal fiber of $A$ is $d-1$ if and only if $A$ is finitely dominated by an analytically ramified stable domain $R$ of embedding dimension $d$ (see Corollary 5.11). In Theorem 5.3 we show that the ring $R$ arises via the construction of Heinzer, Rotthaus, and Sally in a natural way from a prime ideal $P$ of $\widehat{A}$ such that $\widehat{A} / P$ has dimension 1 and $P \cap A=0$, and such that the embedding dimension of the ring $R$ is one more than the embedding dimension of the ring $\widehat{A}_{P}$. Thus, when $A$ is excellent or, more generally, a $G$-ring, then this local ring $\widehat{A}_{P}$ is a regular local ring, and hence the embedding dimension of $\widehat{A}_{P}$ is $d-1$. We obtain from this observation a bound on the embedding dimensions of the analytically ramified local Noetherian stable rings that finitely dominate $A$ (see Corollary 5.6).

The main results regarding the connection between stable rings and the generic formal fiber are in Sections 5 and 6 , but since our main focus is on 1-dimensional stable rings, and since stable rings are also of interest in nonNoetherian commutative ring theory (for some recent examples, see [4], [6], [9], [19], [28], [32], [33], [34]), we include in Sections 3 and 4 characterizations of these rings in terms of their normalization and completion; for more in this vein, see also [26].

Notation and terminology. All rings are commutative with identity. We use the following standard notation throughout the article. For a ring $R$, we denote by $\bar{R}$ the integral closure of $R$ in its total ring quotients. Thus, $\bar{R}$ is the normalization of $R$. The ring $R$ has finite normalization if $\bar{R}$ is a finite 
$R$-module. When $R$ is quasi-local with maximal ideal $M$, we denote by $\widehat{R}$ the completion of $R$ in the $M$-adic topology. The embedding dimension of the quasi-local ring $R$, denoted emb.dim $R$, is the cardinality of a minimal generating set of $M$.

Let $A \subseteq S$ be an extension of rings, and let $L$ be an $S$-module. An $A$-linear derivation $D: S \rightarrow L$ is an $A$-linear mapping with $D(s t)=s D(t)+t D(s)$ for all $s, t \in S$. There exists an $S$-module $\Omega_{S / A}$, as well as an $A$-linear derivation $d_{S / A}: S \rightarrow L$, such that for each $A$-linear derivation $D: S \rightarrow L$, there is a unique $S$-module homomorphism $\alpha: \Omega_{S / A} \rightarrow L$ with $D=\alpha \circ d_{S / A}$ (see $[17$, pp. 191-192]). The module $\Omega_{S / A}$ is the module of Kähler differentials of the extension $A \subseteq S$, and $d_{S / A}$ is the exterior differential of this extension.

\section{$\S 2$. Preliminaries on stable rings}

The terminology of stable ideals originates with Lipman [11] and Sally and Vasconcelos [30]. We leave the motivation for the terminology unexplained here and refer instead to [24] for background on this class of rings. An ideal $I$ of a ring $R$ is stable if it is projective over its ring of endomorphisms. A domain $R$ is stable provided that every nonzero ideal is stable. An ideal $I$ of a quasi-local domain $R$ is stable if and only if $I^{2}=i I$ for some $i \in I$, if and only if $I$ is a principal ideal of $\operatorname{End}(I):=\left(I:_{F} I\right)$, where $F$ is the quotient field of $R$ (see [11] and [25, Lemma 3.1]). It follows from the principal ideal theorem that a Noetherian stable domain has Krull dimension at most 1. If $R$ is a 2-generator domain (meaning every ideal can be generated by two elements), then $R$ is a stable domain (see [2]). Conversely, when $R$ is a Noetherian stable domain with finite normalization, then $R$ is a 2-generator domain (see [3], [29]).

Sally and Vasconcelos [30, Example 5.4] showed that there do exist local Noetherian stable domains without the 2-generator property (and hence without finite normalization). Their example was constructed using derivations and a method of Ferrand and Raynaud and relied on a specific field of characteristic 2. Heinzer, Lantz, and Shah [7, (3.12)] also observed that such examples (again, using this specific field of characteristic 2) could be found for any choice of multiplicity. Note that for a local Noetherian stable ring $R$ with maximal ideal $M$, since $M^{2}=m M$ for some $m \in M$, the multiplicity and embedding dimension of $R$ agree. In this article we see many more such examples of analytically ramified 1-dimensional local stable domains 
and in all possible characteristics. Our focus in this article is on the Noetherian case, but we develop characterizations in Sections 3 and 4 for the general 1-dimensional case as well, since it requires little extra effort. In [27], examples of non-Noetherian 1-dimensional stable domains are given.

To simplify terminology, we say that a domain $R$ is a bad stable domain if it is a quasi-local stable domain with Krull dimension 1 that does not have finite normalization. The domain $R$ is a bad 2-generator ring if $R$ is a 2-generator local ring that does not have finite normalization. These rings are "bad" in the sense of Nagata [21, appendix "Examples of bad Noetherian rings"] because they do not have finite normalization. Thus, a bad Noetherian stable domain is a (necessarily 1-dimensional) analytically ramified local Noetherian stable domain. A bad 2-generator domain is an analytically ramified 2-generator domain.

In later sections we often use the following characterization of bad stable domains, which is a consequence of [25, Corollary 4.3], [22, Corollary 2.5], and [23, Lemma 3.7]. We say that an extension $R \subseteq S$ of rings is quadratic if every $R$-submodule of $S$ containing $R$ is a ring; equivalently, st $\in s R+t R+R$ for all $s, t \in S$.

Proposition 2.1. A quasi-local domain $R$, not a DVR, is a bad stable domain if and only if $\bar{R}$ is a DVR such that $\bar{R} / R$ is a divisible $R$-module and $R \subseteq \bar{R}$ is a quadratic extension.

It is also useful to note that when $R$ is a quasi-local domain and $\bar{R}$ is a DVR, then $\bar{R} / R$ is a divisible $R$-module if and only if $\bar{R}$ tightly dominates $R$. Thus, a quasi-local domain $R$, not a DVR, is a bad stable domain if and only if $R \subseteq \bar{R}$ is a quadratic extension and $\bar{R}$ is a DVR that tightly dominates $R$.

\section{$\S 3$. The completion of a bad stable ring}

Let $R$ be a ring, and let $C$ be a multiplicatively closed set of nonzerodivisors of $R$. An $R$-module $L$ is $C$-divisible if for each $c \in C$ and $\ell \in L$, there exists $\ell^{\prime} \in L$ such that $\ell=c \ell^{\prime}$. The module $L$ is $C$-torsion provided that, for all $\ell \in L$, there exists $c \in C$ such that $c \ell=0$. When $R \subseteq S$ is an extension of rings and $L$ is an $R$-module, we say that $L$ admits an $S$-module structure if there exists an $S$-module structure on $L$ extending its $R$-module structure.

LEMmA 3.1. Let $R \subseteq S$ be an extension of rings, and let $C$ be a multiplicatively closed subset of $R$ consisting of nonzerodivisors of $S$. Suppose that $S / R$ is $C$-divisible, and let $T$ be a $C$-torsion $R$-module. 
(1) The $R$-module $T$ admits an $S$-module structure if and only if for every $t \in T$, it is the case that $\left(0:_{R} t\right) S \cap R=\left(0:_{R} t\right)$.

(2) If $T$ admits an $S$-module structure, then this structure is unique and is given for each $s \in S$ and $t \in T$ by $s \cdot t=r t$, where $r$ is any member of $R$ such that $s-r \in(0: R t) S$ (and such a member $r$ of $R$ must exist).

Proof. (1) If $T$ admits an $S$-module structure $*$, then, using the fact that * extends the $R$-module structure on $T$, it is easy to check that for all $t \in T$, it is the case that $\left(0:_{R} t\right) S \cap R=\left(0::_{R} t\right)$. Conversely, suppose that for all $t \in T$, it is the case that $\left(0:_{R} t\right) S \cap R=\left(0:_{R} t\right)$. Let $s \in S$, and let $t \in T$. Then since $T$ is $C$-torsion, there exists $c \in C$ such that $c t=0$. Thus, since $S=R+c S$, we have that $S=R+\left(0:_{R} t\right) S$, and there exists $r \in R$ such that $s-r \in\left(0:_{R} t\right) S$. We define $s \cdot t=r t$. We omit the calculations, but it is straightforward to check that this defines an $S$-module structure on $T$.

(2) Suppose that $*$ denotes an $S$-module structure on $T$ that extends the $R$-module structure on $T$. We show that the operations $*$ and $\cdot$, where $\cdot$ is defined as in (2), induce the same $S$-module structure on $T$. Let $s \in S$, and let $t \in T$. Since $T$ is $C$-torsion, there exists $c \in C$ such that $c t=0$. Thus, since by assumption $S=R+c S$, we may choose $r \in R$ and $\sigma \in S$ such that $s-r=c \sigma$. Then, using the fact that $*$ extends the $R$-module structure on $T$, we have $s * t=(r+c \sigma) * t=1 * r t+\sigma *(c t)=r t$, which proves that $s * t=s \cdot t$.

Recall from Section 2 that a ring extension $R \subseteq S$ is quadratic if every $R$-submodule of $S$ containing $R$ is a ring.

LEMmA 3.2. Let $R \subseteq S$ be an extension of rings, and suppose that there exists a multiplicatively closed subset $C$ of $R$ consisting of nonzerodivisors in $S$ such that $S / R$ is $C$-torsion and $C$-divisible. Then the following statements are equivalent.

(1) $R \subseteq S$ is a quadratic extension of rings.

(2) For all $s \in S,\left(R:_{R} s\right)=\left(R:_{R} s\right) S \cap R$.

(3) $S / R$ admits an $S$-module structure.

(4) There exist an $S$-module $T$ and a derivation $D: S \rightarrow T$ with $R=\operatorname{Ker} D$.

(5) The mapping $S / R \rightarrow \Omega_{S / R}$ induced by $d_{S / R}$ is an isomorphism of $R$-modules.

(6) For all $c \in C,(R \cap c S)^{2} \subseteq c R$.

Proof. $(1) \Rightarrow(2)$ Let $s \in S$, and suppose that $x \in(R: R s) S \cap R$. We claim that $x \in\left(R:_{R} s\right)$. Since $S / R$ is $C$-torsion, there exists $c \in C$ such that $c s \in R$. 
Also, since $S=R+c S$, we have $\left(R:_{R} s\right) S=\left(R:_{R} s\right)+c\left(R:_{R} s\right) S \subseteq\left(R:_{R}\right.$ $s)+c S$, so that there exist $a \in\left(R:_{R} s\right)$ and $\sigma \in S$ such that $x=a+c \sigma$. By (1), $s \sigma \in s R+\sigma R+R$, so $c s \sigma \in c s R+c \sigma R+c R$. Thus, since $c s \in R$, $c \sigma=x-a \in R$, and $c \in R$, it follows that $c \sigma \in\left(R:_{R} s\right)$. By assumption, $a \in(R: R s)$, so we conclude that $x=a+c \sigma \in(R: R s)$, which proves (2).

$(2) \Rightarrow(3)$ Since $S / R$ is $C$-divisible and $C$-torsion, and since by $(2),\left(R:_{R}\right.$ $s) S \cap R=\left(R:_{R} s\right)$ for all $s \in S$, we have by Lemma 3.1(1) that $S / R$ admits an $S$-module structure.

(3) $\Rightarrow(4)$ Define a mapping $D: S \rightarrow S / R$ by $D(s)=s+R$ for all $s \in S$. By (3), $S / R$ has an $S$-module structure, which by Lemma 3.1(2) must be given by the operation - defined in the lemma. We claim that with this $S$-module structure on $S / R, D$ is a derivation, for once this is proved, (4) follows at once. Let $s_{1}, s_{2} \in S$. Then since $S / R$ is $C$-divisible and $C$-torsion, there exist $r_{1}, r_{2} \in R, \sigma_{1}, \sigma_{2} \in S, c_{1} \in\left(R:_{R} s_{2}\right)$, and $c_{2} \in\left(R:_{R} s_{1}\right)$ such that $s_{1}=r_{1}+c_{1} \sigma_{1}$ and $s_{2}=r_{2}+c_{2} \sigma_{2}$. Then applying the definition of $\cdot$ we have

$$
s_{1} \cdot D\left(s_{2}\right)+s_{2} \cdot D\left(s_{1}\right)=s_{1} \cdot\left(s_{2}+R\right)+s_{2} \cdot\left(s_{1}+R\right)=r_{1} s_{2}+r_{2} s_{1}+R .
$$

Hence, to prove that $D\left(s_{1} s_{2}\right)=s_{1} \cdot D\left(s_{2}\right)+s_{2} \cdot D\left(s_{1}\right)$, it suffices to show that $D\left(s_{1} s_{2}\right)=r_{1} s_{2}+r_{2} s_{1}+R$. Now

$$
\begin{aligned}
D\left(s_{1} s_{2}\right) & =s_{1} s_{2}+R=\left(r_{1}+c_{1} \sigma_{1}\right)\left(r_{2}+c_{2} \sigma_{2}\right)+R \\
& =r_{1} c_{2} \sigma_{2}+r_{2} c_{1} \sigma_{1}+c_{1} c_{2} \sigma_{1} \sigma_{2}+R \\
& =r_{1}\left(s_{2}-r_{2}\right)+r_{2}\left(s_{1}-r_{1}\right)+c_{1} c_{2} \sigma_{1} \sigma_{2}+R \\
& =r_{1} s_{2}+r_{2} s_{1}+c_{1} c_{2} \sigma_{1} \sigma_{2}+R .
\end{aligned}
$$

Therefore, we need only verify that $c_{1} c_{2} \sigma_{1} \sigma_{2} \in R$. To this end, observe that since $c_{2} \in\left(R:_{R} s_{1}\right)$, then $0+R=c_{2} s_{1}+R=c_{2}\left(r_{1}+c_{1} \sigma_{1}\right)+R=c_{2} c_{1} \sigma_{1}+R$, so $c_{1} c_{2} \sigma_{1} \in R$. Hence, using the fact that · extends the $R$-module structure on $S / R$, as well as the fact that $c_{1}, c_{2}, c_{1} s_{2}$, and $c_{1} c_{2} \sigma_{1}$ are all members of $R$, we have

$$
\begin{aligned}
0+R & =\sigma_{1} \cdot\left(c_{1} s_{2}+R\right)=c_{1} \sigma_{1} \cdot\left(r_{2}+c_{2} \sigma_{2}+R\right) \\
& =c_{1} \sigma_{1} \cdot\left(c_{2} \sigma_{2}+R\right)=c_{1} c_{2} \sigma_{1} \cdot\left(\sigma_{2}+R\right)=c_{1} c_{2} \sigma_{1} \sigma_{2}+R .
\end{aligned}
$$

Therefore, $c_{1} c_{2} \sigma_{1} \sigma_{2} \in R$, which proves that $D\left(s_{1} s_{2}\right)=s_{1} \cdot D\left(s_{2}\right)+s_{2} \cdot D\left(s_{1}\right)$. Clearly, $D\left(s_{1}+s_{2}\right)=D\left(s_{1}\right)+D\left(s_{2}\right)$, so $D$ is a derivation. 
$(4) \Rightarrow(1)$ Let $x, y \in S$. We show that $x y \in x R+y R+R$. Since $S / R$ is $C$-torsion, there exists $c \in C$ such that $c x, c y \in R$. Thus, since $c \in R=$ Ker $D$, we have $c D(x)=D(c x)=0$ and $c D(y)=D(c y)=0$. Also, since $S / R$ is $C$-divisible, there exist $a, b \in R$ such that $x+a, y+b \in c S$. Hence, $(x+a) D(y)=0$ and $(y+b) D(x)=0$. Therefore, since also $D(a)=D(b)=0$, we have

$$
\begin{aligned}
D(x y+y a+x b) & =D(x y)+D(y a)+D(x b) \\
& =x D(y)+y D(x)+a D(y)+b D(x) \\
& =(x+a) D(y)+(y+b) D(y)=0 .
\end{aligned}
$$

Thus, $x y+y a+x b \in \operatorname{Ker} D=R$, whence $x y \in x R+y R+R$.

$(4) \Rightarrow(5)$ Assuming (4), there exists an $S$-module homomorphism $\alpha$ : $\Omega_{S / R} \rightarrow T$ such that $\alpha \circ d_{S / R}=D$ (see Section 1). Thus, $\operatorname{Ker} d_{S / R} \subseteq \operatorname{Ker} D=$ $R$. But $d_{S / R}$ is an $R$-linear derivation, so it must be that $\operatorname{Ker} d_{S / R}=R$. Thus, to verify (5), it suffices to show that $d_{S / R}$ is an onto mapping. To this end, note that since $d_{S / R}(S)$ generates $\Omega_{S / R}$ as an $S$-module, there exist $s_{1}, \ldots, s_{n}, x_{1}, \ldots, x_{n} \in S$ such that $y=\sum_{i=1}^{n} s_{i} d\left(x_{i}\right)$. Choose $c \in C$ such that $c x_{1}, \ldots, c x_{n} \in R$. Then since $S=\operatorname{Ker} d_{S / R}+c S$, we may for each $i$ write $s_{i}=$ $a_{i}+c \sigma_{i}$, where $a_{i} \in \operatorname{Ker} d_{S / R}$ and $\sigma_{i} \in S$. Thus, since $a_{1}, \ldots, a_{n} \in \operatorname{Ker} d_{S / R}$, we have

$$
y=\sum_{i} d_{S / R}\left(a_{i} x_{i}\right)+\sum_{i} \sigma_{i} c d_{S / R}\left(x_{i}\right)=d_{S / R}\left(\sum_{i} a_{i} x_{i}\right) .
$$

Therefore, $d_{S / R}$ maps onto $\Omega_{S / R}$, and $\Omega_{S / R}$ and $S / R$ are isomorphic as $R$-modules.

$(5) \Rightarrow(3)$ Since $\Omega_{S / R}$ is an $S$-module, this is clear.

$(1) \Rightarrow(6)$ Let $c \in C$, and let $s_{1}, s_{2} \in S$ such that $c s_{1}, c s_{2} \in R$. It suffices to show that $c^{2} s_{1} s_{2} \in c R$. By (1), $s_{1} s_{2} \in s_{1} R+s_{2} R+R$, so that $c^{2} s_{1} s_{2} \in$ $c\left(c s_{1}\right) R+c\left(c s_{2}\right) R+c^{2} R \subseteq c R$, as claimed.

$(6) \Rightarrow(2)$ Let $s \in S$, and let $x \in(R: R s) S \cap R$. Since $S / R$ is $C$-torsion, there exists $c \in C \cap\left(R:_{R} s\right)$. Also, since $S / R$ is $C$-divisible, we have $S=$ $R+c S$, and hence $\left(R:_{R} s\right) S=\left(R:_{R} s\right)+c S$. Thus, there exist $a \in\left(R:_{R} s\right)$ and $\sigma \in S$ such that $x=a+c \sigma$, and in order to show that $x \in\left(R:_{R} s\right)$, it suffices to prove that $c \sigma s \in R$. Since $c \sigma=x-a \in R$ and $c s \in R$, we have $(c \sigma)(c s) \in(c S \cap R)^{2}$, so that by (6), $c^{2} \sigma s \in c R$. Thus, since $c$ is a nonzerodivisor in $S, c \sigma s \in R$, as claimed. 
Theorem 3.4 shows that the completion of a bad stable domain has a prime ideal whose square is 0 and whose residue ring is a DVR. Ideals in such rings have principal reductions of reduction number at most 1 , as follows.

Lemma 3.3. Let $N$ be an ideal of the ring $R$ such that $N^{2}=0$. If $I$ is an ideal of $R$ whose image in $R / N$ is a principal ideal generated by $x+N$ for some $x \in I$, then $I^{2}=x I$.

Proof. To prove that $I^{2}=x I$, it suffices to show that for all $y, z \in I, y z \in$ $x(x, y, z) R=\left(x^{2}, x y, x z\right) R$. Let $y, z \in I$. Write $y=x r_{1}+n_{1}$ and $z=x r_{2}+n_{2}$ for some $r_{1}, r_{2} \in R$ and $n_{1}, n_{2} \in N$. Observe that since $N^{2}=0$, we have

$$
y z=x^{2} r_{1} r_{2}+x r_{1} n_{2}+x r_{2} n_{1}, \quad x y=x^{2} r_{1}+x n_{1}, \quad x z=x^{2} r_{2}+x n_{2} .
$$

The following calculation now shows that $y z \in\left(x^{2}, x y, x z\right) R$ :

$$
\begin{aligned}
-r_{1} r_{2}\left(x^{2}\right)+r_{2}(x y)+r_{1}(x z) & =-r_{1} r_{2} x^{2}+r_{2}\left(x^{2} r_{1}+x n_{1}\right)+r_{1}\left(x^{2} r_{2}+x n_{2}\right) \\
& =-r_{1} r_{2} x^{2}+r_{1} r_{2} x^{2}+r_{2} x n_{1}+r_{1} r_{2} x^{2}+r_{1} x n_{2} \\
& =r_{1} r_{2} x^{2}+r_{2} x n_{1}+r_{1} x n_{2}=y z .
\end{aligned}
$$

Thus, $y z \in x(x, y, z) R \subseteq x I$, which proves that $I^{2}=x I$.

We give in the next theorem the characterization of the completion of a bad stable ring, but there is a small subtlety in how it is phrased. The theorem is proved for not-necessarily-Noetherian rings, and because of this the domain $R$ need not be separated in the m-adic topology. (A bad stable domain is easily seen to be separated, but in the converse, in showing that the given domain is a bad stable domain, separation is needed to guarantee that $R \rightarrow \widehat{R}$ is a flat embedding.) Thus, we state the theorem for the completion of $R$ in the ideal topology (or $R$-topology): $\widetilde{R}:=\lim _{\leftarrow} R / r R$, where $r$ ranges over all nonzero elements of $R$. (For properties and applications of this completion, see [5], [15], [14].) When $R$ is a 1-dimensional Noetherian ring, which is the main case of interest in this article, then $\widetilde{R}$ is isomorphic as an $R$-algebra to $\widehat{R}$, and we deduce in Corollary 3.5 an $\mathfrak{m}$-adic version of the theorem in the Noetherian case.

TheOREM 3.4. A 1-dimensional quasi-local domain is a bad stable ring if and only if there is a nonzero prime ideal $P$ of $\widetilde{R}$ such that $P^{2}=0$ and $\widetilde{R} / P$ is a $D V R$. 
Proof. Let $R$ be a bad stable ring, and let $r$ be a nonzero nonunit in $R$. Then since $R$ has dimension $1, Q:=R[1 / r]$ is the quotient field of $R$, and hence $Q$ is a countably generated $R$-module. By [5, Lemma VI.2.6, p. 203], this in turn implies that $Q$ has projective dimension 1 as an $R$-module. Since we are working with the completion of $R$ in the ideal topology, and $Q$ has projective dimension 1 , there is a surjective ring homomorphism $\psi: \widetilde{R} \rightarrow$ $(\bar{R})^{\sim}$, where $(\bar{R})^{\sim}$ is the completion of $\bar{R}$ in the ideal topology of $\bar{R}$ (see $[15$, Theorem 2.9, p. 21]). Since $\bar{R}$ is a DVR that is integral over $R$, it follows that the $R$-topology and $\bar{R}$-topology on $\bar{R}$ agree, so with $S:=\lim _{\leftarrow} \bar{R} / r \bar{R}$, where $r$ ranges over the nonzero elements of $R$, the canonical mapping $\phi: \widetilde{R} \rightarrow S$ is a surjection. Now $S$, as completion of a DVR, is a DVR, and since $R$ is not a DVR, $P:=\operatorname{Ker} \phi \neq 0$, so we need only show that $P^{2}=0$. To this end, let $x, y \in P$. Write $x=\left\langle x_{r}+r R\right\rangle$ and $y=\left\langle y_{r}+r R\right\rangle$. (We are viewing $\widetilde{R}$ as a subring of $\prod_{r} R / r R$ and $S$ as a subring of $\prod_{R} \bar{R} / r \bar{R}$.) Then $0=\phi(x)=\left\langle x_{r}+r \bar{R}\right\rangle$, so that for each $r, x_{r} \in r \bar{R} \cap R$. Similarly, $y_{r} \in r \bar{R} \cap R$, and hence by Lemma 3.2(6), $x_{r} y_{r} \in(r \bar{R} \cap R)^{2} \subseteq r R$, proving that $x y=0$, and hence that $P^{2}=0$.

Conversely, suppose that there is a nonzero prime ideal $P$ of $\widetilde{R}$ such that $P^{2}=0$ and $\widetilde{R} / P$ is a DVR. If $I$ is a nonzero proper ideal of $R$, then since the image of $I \widetilde{R}$ in $\widetilde{R} / P$ is a principal ideal, we have by Lemma 3.3 that there exists $x \in I \widetilde{R}$ such that $I^{2} \widetilde{R}=x I \widetilde{R}$. We show that this implies that there exists $i \in I$ such that $I^{2}=i I$. With $\lambda: R \rightarrow \widetilde{R}$ the canonical map, the $R$-module $\widetilde{R} / \lambda(R)$ is divisible (see [15, Theorem 2.1, p. 11]). Thus, $x \in \lambda(R)+x^{2} \widetilde{R}$, and there exist $y \in \widetilde{R}$ and $i \in R$ with $x(1-x y)=\lambda(i)$. Since $x$ is not a unit in the quasi-local $\operatorname{ring} \widetilde{R}$, it follows that $x \widetilde{R}=i \widetilde{R}$. Moreover, since $\widetilde{R}$ is a flat $R$-module (see [15, Corollary 2.6]), $i \in \lambda^{-1}(I \widetilde{R})=I$. This shows that there exists $i \in I$ such that $I^{2} \widetilde{R}=i I \widetilde{R}$. Again by flatness, $I^{2}=i I$. Thus, $I$ is a stable ideal of $R$, and $R$ is a stable domain. Moreover, $R$ is a bad stable domain. Indeed, suppose that $R$ has finite normalization. Then $R$ is a 1-dimensional local Noetherian domain, so that $\widetilde{R}=\widehat{R}$. Thus, since $R$ has finite normalization, then $\widetilde{R}$ is reduced, contrary to the fact that $P$ is a nonzero ideal of $\widetilde{R}$ with $P^{2}=0$.

Restricting to Noetherian rings, the $\mathfrak{m}$-adic and ideal completions agree, so we obtain the following.

Corollary 3.5. A local Noetherian domain is a bad stable domain if and only if there is a nonzero prime ideal $P$ of $\widehat{R}$ such that $P^{2}=0$ and $\widehat{R} / P$ is a DVR. 


\section{$\S 4$. Characterizations of bad stable rings}

An $R$-module $K$ is uniserial if the set of all $R$-submodules of $K$ is linearly ordered with respect to inclusion.

LEMMA 4.1. Let $R \subseteq S$ be an integral extension of rings, with $S$ a quasilocal ring. If $S / R$ is an Artinian uniserial $R$-module, then $R \subseteq S$ is a quadratic extension.

Proof. Let $\mathcal{S}$ be the set of all $R$-submodules of $S$ containing $R$, and let $\mathcal{R}$ be the set of all $B \in \mathcal{S}$ such that $B$ is a ring. We claim that $\mathcal{S}=\mathcal{R}$, and hence that $R \subseteq S$ is a quadratic extension. Since $S / R$ is Artinian and uniserial, the set $\mathcal{S}$ is well ordered with respect to inclusion, so we may use transfinite induction to show that $\mathcal{S}=\mathcal{R}$. In particular, to prove that $\mathcal{S}=\mathcal{R}$, it suffices to show that for each $F \in \mathcal{S}$,

$$
\{E \in \mathcal{S}: E \subsetneq F\} \subseteq \mathcal{R} \Rightarrow F \in \mathcal{R} .
$$

Let $F \in \mathcal{S}$, and suppose that $\{E \in \mathcal{S}: E \subsetneq F\} \subseteq \mathcal{R}$. Let $E^{\prime}$ be the union of all the $E \in \mathcal{S}$ such that $E \subsetneq F$. Then since each such $E$ is a ring and $\mathcal{S}$ is a chain, $E^{\prime}$ is also a ring. Thus, if $E^{\prime}=F$, then the claim that $F \in \mathcal{R}$ is proved. Otherwise, suppose that $E^{\prime} \subsetneq F$. Since $E^{\prime}$ is a ring and $S$ is quasi-local and integral over $R$, it follows that $E^{\prime}$ has a unique maximal ideal $M$. Now $F / E^{\prime}$ is necessarily a simple $E^{\prime}$-module, so $M F \subseteq E^{\prime}$. In fact, $M F \subseteq M$, since otherwise the maximality of $M$ in $E^{\prime}$ implies that $1 \in M F \subseteq M S$, a contradiction to the fact that $S$ is integral over $E^{\prime}$. Therefore, $F \subseteq\left(M:_{S} M\right)$. Yet $F / E^{\prime} \subseteq\left(M:_{S} M\right) / E^{\prime}$ is a containment of vector spaces over $R /(M \cap R)$, so the fact that $S / R$, and hence $S / E^{\prime}$, is a uniserial $R$-module implies that $(M: S M) / E^{\prime}$ is a simple $R$-module, and this forces $F=(M: S M)$. Therefore, $F$ is a ring, and $F \in \mathcal{R}$, which proves that $\mathcal{R}=\mathcal{S}$. This shows that $R \subseteq S$ is a quadratic extension.

THEOREM 4.2. The following statements are equivalent for a quasi-local domain $R$ with quotient field $F$.

(1) $R$ is a bad stable domain.

(2) $\bar{R}$ is a $D V R$, and $\bar{R} / R \cong \bigoplus_{i \in I} F / \bar{R}$ as $R$-modules for some index set $I$.

(3) $\bar{R}$ is a DVR, and $\bar{R} / R$ is a direct sum of divisible Artinian uniserial $R$-modules.

Proof. (1) $\Rightarrow(2)$ Since by Proposition $2.1 \bar{R} / R$ is a torsion divisible $R$-module and $R \subseteq \bar{R}$ is a quadratic extension, we may apply Lemma 3.2 
to obtain that $\bar{R} / R$ is an $\bar{R}$-module. Now since $\bar{R}$ and $R$ share the same quotient field, as a divisible $R$-module, $\bar{R} / R$ is also a divisible $\bar{R}$-module. Also, by Proposition $2.1, \bar{R}$ is a DVR, and hence every divisible $\bar{R}$-module is an injective $\bar{R}$-module. In particular, $\bar{R} / R$ is an injective $\bar{R}$-module. Let $N$ denote the maximal ideal of $\bar{R}$. Since $\bar{R}$ is a Noetherian domain, every injective $\bar{R}$-module is a direct sum of indecomposable $\bar{R}$-modules (see [12, Theorem 2.5]), and since also $\bar{R}$ is a DVR, a torsion $\bar{R}$-module is injective and indecomposable if and only if it is isomorphic to $F / \bar{R}$. (For example, combine [13, Theorem 4] and [15, Theorem 4.5].) Therefore, since $\bar{R} / R$ is a torsion injective $\bar{R}$-module, we have that $\bar{R} / R$ is isomorphic as an $\bar{R}$-module, and hence an $R$-module, to a direct sum of copies of $F / \bar{R}$.

$(2) \Rightarrow(3)$ By $(2), \bar{R} / R$ is a direct sum of $R$-modules, say, $\bar{R} / R=\bigoplus_{i \in I} B_{i} /$ $R$, where each $B_{i} / R \cong F / \bar{R}$ as $R$-modules. Since $\bar{R}$ is a DVR, $F / \bar{R}$ is a divisible Artinian uniserial $\bar{R}$-module. Thus, to show that for each $i, B_{i} / R$ is a divisible Artinian uniserial $R$-module, it suffices to show that every $R$-submodule of $F / \bar{R}$ is also an $\bar{R}$-submodule. Let $A / \bar{R}$ be an $R$-submodule of $F / \bar{R}$. We show that $A \bar{R} \subseteq A$. Let $a \in A$, and let $x \in \bar{R}$. Since $F / \bar{R}$ is a torsion $R$-module, there exists $0 \neq r \in R$ such that $r a \in \bar{R}$. Since $\bar{R} / R$ is divisible, there exist $s \in R$ and $y \in \bar{R}$ with $x=s+r y$. Therefore, since $\bar{R} \subseteq A$, then $x a=s a+(r a) y \in A$, which proves that every $R$-submodule of $F / \bar{R}$ is an $R$-module.

$(3) \Rightarrow(1)$ Write $\bar{R} / R=\bigoplus_{i \in I} B_{i} / R$, where for each $i \in I, B_{i} / R$ is a divisible Artinian uniserial $R$-module. Fix $i \in I$, and let $B=\sum_{j \neq i} B_{j}$. We claim first that $B_{i} / R$ is an $\bar{R}$-module. Indeed, since $\bar{R}=B_{i}+B$ and $R=B_{i} \cap B$, we have $B_{i} / R \cong\left(B_{i}+B\right) / B=\bar{R} / B$ as $R$-modules. Now $B_{i} / R$ is by assumption a divisible Artinian uniserial $R$-module, so $\bar{R} / B$ is also a divisible Artinian uniserial $R$-module, and hence also a divisible Artinian uniserial $B$-module (where divisibility as a $B$-module follows from the fact that $B / R$ is a torsion $R$-module). Observe that $B$ is a ring. Let $a, b \in B$. Then there exists $0 \neq r \in R$ such that $r b \in R$. Since $B / R$ is a divisible $R$-module, there exist $c \in B$ and $s \in R$ with $a=r c+s$. Hence, $a b=(r c+s) b=(r b) c+s b \in B$, proving that $B$ is a ring. Therefore, by Lemma $4.1, B \subseteq \bar{R}$ is a quadratic extension, and hence by Lemma $3.2, \bar{R} / B$ is an $\bar{R}$-module. But then, since $B_{i} / R \cong \bar{R} / B$, we have that $B_{i} / R$ admits an $\bar{R}$-module structure. Therefore, $\bar{R} / R$, as a direct sum of the $B_{i} / R$, also admits an $\bar{R}$-module structure, and by Lemma 3.2, $R \subseteq \bar{R}$ is a quadratic extension. Thus, since $\bar{R}$ is a DVR, we have by Proposition 2.1 that $R$ is a bad stable domain. 
Corollary 4.3. Let $R$ be a quasi-local domain with quotient field $F$, and let $n>1$. Then the following statements are equivalent.

(1) $R$ is a bad Noetherian stable domain of embedding dimension $n$.

(2) $\bar{R}$ is a DVR, and $\bar{R} / R$ is a direct sum of $n-1$ divisible Artinian uniserial $R$-modules.

(3) $\bar{R}$ is a DVR, and $\bar{R} / R \cong \bigoplus_{i=1}^{n-1} F / \bar{R}$ as $R$-modules.

Proof. (1) $\Rightarrow(2)$ Assume (1), and let $M$ denote the maximal ideal of $R$. By Theorem $4.2, \bar{R}$ is a DVR and $\bar{R} / R$ is a direct sum of divisible Artinian uniserial $R$-modules, say, $\bar{R} / R=\bigoplus_{i \in I} B_{i} / R$, where each $B_{i} / R$ is a divisible Artinian uniserial $R$-module. For each $i$, since $B_{i} / R$ is Artinian, there exists an $R$-submodule $D_{i}$ of $B_{i}$ such that $R \subseteq D_{i} \subseteq B_{i}$ and $D_{i} / R$ is a simple $R$-module. Hence, for each $i, M D_{i} \subseteq R$, and we have $D_{i} \subseteq M^{-1}:=\left(R:_{F}\right.$ $M)$. We claim that $M^{-1} / R=\bigoplus_{i \in I} D_{i} / R$. For each $i \in I$, let $\pi_{i}$ be the projection of $\bar{R} / R$ onto $B_{i} / R$. Then for each $i, D_{i} / R \subseteq \pi_{i}\left(M^{-1} / R\right) \subseteq B_{i} / R$. Now $\pi_{i}\left(M^{-1} / R\right)$ is an $R / M$-vector space, so since $B_{i} / R$ is uniserial, it must be that $\pi_{i}\left(M^{-1} / R\right)$ has dimension 1 as an $R / M$-vector space and hence is equal to $D_{i} / R$. Therefore, $\bigoplus_{i \in I} D_{i} / R \subseteq M^{-1} / R \subseteq \bigoplus_{i \in I} D_{i} / R$, and since each $D_{i} / R$ is a simple $R$-module, we conclude that the vector space dimension of $M^{-1} / R$ and the cardinality $|I|$ of $I$ are the same. Now since $R$ is not a DVR, we have $M M^{-1}=M$; then $M^{-1}=\left(M:_{F} M\right)$. The fact that $M$ is stable then implies that $M=m M^{-1}$ for some $m \in M$, and hence $M / M^{2}=m M^{-1} / m M \cong M^{-1} / M$. The embedding dimension $n$ of $R$ is thus the same as the dimension of the $R / M$-vector space $M^{-1} / M$. Therefore, the dimension of the $R / M$-vector space $M^{-1} / R$ is $n-1$, which forces $|I|=n-1$.

$(2) \Rightarrow(1)$ By Theorem 4.2, $R$ is a bad stable domain. Thus, as we saw above in the conclusion of the proof of $(1) \Rightarrow(2)$, the number of generators needed for $M$ is one more than the dimension of $M^{-1} / R$. Moreover, as in the proof of $(1) \Rightarrow(2)$, this dimension is the same as the number of divisible uniserial Artinian $R$-modules in the decomposition of $\bar{R} / R$, which by assumption is $n-1$.

$(2) \Leftrightarrow(3)$ This is clear in view of the proof of Theorem 4.2.

The last theorem of this section shows that wherever a bad stable domain $R$ can be found, it is possible to find bad Noetherian stable overrings of $R$ with prescribed embedding dimension, as long as that embedding dimension does not surpass the embedding dimension of $R$. 
THEOREM 4.4. Let $R$ be a bad stable domain of embedding dimension $n$ (where possibly $n$ is infinite). Then we have the following.

(1) If $n$ is finite (equivalently, if $R$ is Noetherian), then for every ring $S$ with $R \subseteq S \subsetneq \bar{R}$, the ring $S$ is a bad Noetherian stable domain with embedding dimension at most $n$.

(2) For every integer $k$ with $2 \leq k \leq n$, there is a bad Noetherian stable domain $S$ of embedding dimension $k$ with $R \subseteq S \subseteq \bar{R}$ and such that $S / R$ is isomorphic to a direct sum of copies of $F / \bar{R}$.

Proof. (1) Let $S$ be a ring with $R \subseteq S \subsetneq \bar{R}$. Since $S \subseteq \bar{R}$ is a quadratic extension, $\bar{R}$ is a DVR, and $\bar{R} / S$ is a divisible $S$-module, Proposition 2.1 implies that $S$ is a bad stable domain. Since $R$ is a 1-dimensional local Noetherian domain, the multiplicity of every local overring of $R$ is bounded above by the multiplicity of $R$ (see [20, Theorem 2.1]). Since for a stable ring, embedding dimension and multiplicity agree, statement (1) now follows.

(2) Assume that $2 \leq k<n$. Since $R \subseteq \bar{R}$ is a quadratic extension, Theorem 4.2 implies that there exist $R$-subalgebras $S_{1}, \ldots, S_{k-1}, S$ of $\bar{R}$ such that

$$
\bar{R} / R=S_{1} / R \oplus \cdots \oplus S_{k-1} / R \oplus S / R,
$$

where $S / R$ is isomorphic to a direct sum of copies of $F / \bar{R}$, and for each $i, S_{i} / R \cong F / \bar{R}$. We claim that $S$ is a bad Noetherian stable domain with embedding dimension $k$. Now

$$
\bar{R} / S \cong(\bar{R} / R) /(S / R) \cong S_{1} / R \oplus \cdots \oplus S_{k-1} / R \cong \bigoplus_{i=1}^{k-1} F / \bar{R}
$$

where these isomorphisms are as $R$-modules. Thus, there is an isomorphism of $R$-modules

$$
\alpha: \bar{R} / S \rightarrow \bigoplus_{i=1}^{k-1} F / \bar{R}
$$

To prove that $S$ is a bad Noetherian stable domain with embedding dimension $k$, it suffices by Corollary 4.3 to show that $\alpha$ is an $S$-module homomorphism. Now $\alpha$ induces an $\bar{R}$-module structure on $\bar{R} / S$, which by Lemma 3.1 is the unique $\bar{R}$-module structure extending the $R$-module structure on $\bar{R} / S$ and must be given as in the lemma. Thus, we need only verify that this $\bar{R}$-module structure extends the $S$-module structure on $\bar{R} / S$. Let $s \in S$, and let $v \in \bar{R}$. Then as in Lemma 3.1, $s \cdot(v+S)=r v+S$, where $s-r \in\left(S:_{R} v\right) \bar{R}$. 
But $S \subseteq \bar{R}$ is a quadratic extension, so by Lemma 3.2, $\left(S:_{R} v\right) \bar{R} \cap S \subseteq$ $\left(S:_{S} v\right) \bar{R} \cap S=\left(S:_{S} v\right)$, and hence $s-r \in\left(S:_{S} v\right)$. This then implies that $s v+S=r v+S$, which proves that the $\bar{R}$-module structure on $\bar{R} / S$ extends the $S$-module structure. Hence, $S$ is a bad Noetherian stable domain of embedding dimension $k$.

\section{$\S 5 . \quad$ Representation and embedding dimension of stable rings}

Let $A$ be a local Noetherian domain with maximal ideal $\mathfrak{m}$, and suppose that $I$ is an ideal of $\widehat{A}$ such that every associated prime $P$ of $I$ satisfies $A \cap P=0$, where $A$ is identified with its image in $\widehat{A}$. Then, since $I \cap A=$ 0 , the canonical mapping $A \rightarrow \widehat{A} / I$ is an embedding, and we can identify $A$ with its image in $\widehat{A} / I$. Under this identification, since the associated primes of $I$ contract to 0 in $A$, it follows that the nonzero elements of $A$ are nonzerodivisors in $\widehat{A} / I$. Therefore, the quotient field $F$ of $A$ embeds into the total quotient ring of $\widehat{A} / I$, and hence we may consider the $\operatorname{ring} F \cap(\widehat{A} / I)$. More precisely,

$$
F \cap(\widehat{A} / I)=\left\{\frac{a}{b} \in F: a, b \in A, b \neq 0 \text { and } a \in b \widehat{A}+I\right\} .
$$

We show in Theorem 5.3 that by choosing $I$ appropriately we obtain bad Noetherian stable domains birationally dominating $A$.

Our theorem depends on the following lemma, due to Heinzer, Rotthaus, and Sally in [8]. The result we state here is weaker than theirs except that the last assertion of the lemma is stated there as $\widehat{A} / I \cong \widehat{R}$. However, the stronger form that we have used asserting the surjection of the canonical mapping is justified by the proof in [8].

Lemma 5.1 ([8, Corollary 1.27]). Let $A$ be a local Noetherian domain with quotient field $F$, and let $I$ be an ideal of $\widehat{A}$ with the property that each associated prime $P$ of I satisfies $P \cap A=0$. If the Krull dimension of $\widehat{A} / I$ is 1 , then $R:=F \cap(\widehat{A} / I)$ is a Noetherian domain of Krull dimension 1 , and the canonical mapping $\phi: \widehat{A} \rightarrow \widehat{R}$ is a surjection having kernel $I$.

The following lemma is an application of a standard fact about finite generation of modules over complete local rings.

Lemma 5.2. Let $A$ be a local Noetherian domain with maximal ideal $\mathfrak{m}$. If $R$ is a quasi-local domain of Krull dimension 1 that finitely dominates $A$, then $R$ is a Noetherian domain. If also $R$ tightly dominates $A$, then the 
canonical homomorphism $\widehat{A} \rightarrow \widehat{R}$ is a surjection, where $\widehat{R}$ is the completion of $\widehat{R}$ in the $\mathfrak{m}$-adic topology.

Proof. Note that $\mathfrak{m} \widehat{A} \cdot \widehat{R}=\mathfrak{m} \widehat{R}$ and that $\widehat{R}$ is separated in the $\mathfrak{m} \widehat{A}$-adic topology. Now since $R / \mathfrak{m} R \cong \widehat{R} / \mathfrak{m} \widehat{R}$ and $R / \mathfrak{m} R$ is finite over $A$, there exist elements $\omega_{1}, \ldots, \omega_{n} \in \widehat{R}$ such that $\widehat{R} / \mathfrak{m} \widehat{R}$ is generated as an $\widehat{A}$-module by the images of these elements. Thus, since $\widehat{A}$ is complete in the $\mathfrak{m} \widehat{A}$-adic topology and the $\widehat{A}$-module $\widehat{R}$ is complete and separated in the $\mathfrak{m} \widehat{A}$-adic topology, $\widehat{R}$ is generated as an $\widehat{A}$-module by $\omega_{1}, \ldots, \omega_{n}$ (see $[17$, Theorem 8.4 , p. 58]). Hence, since $\widehat{A}$ is a Noetherian ring, so is the finite $\widehat{A}$-module $\widehat{R}$. In particular, if $M$ denotes the maximal ideal of $R$, then $M \widehat{R}$ is a finitely generated ideal of $\widehat{R}$. Let $0 \neq r \in M$. Then since $R$ has Krull dimension 1 , there exists $i>0$ such that $\mathfrak{m}^{i} \subseteq r R$. Therefore, since $\widehat{R} / \mathfrak{m}^{i} \widehat{R}$ is isomorphic as an $R$-algebra to $R / \mathfrak{m}^{i} R$, it follows that $\widehat{R} / r \widehat{R}$ and $R / r R$ are isomorphic as $R$-algebras. Consequently, since the $R$-module $M \widehat{R} / r \widehat{R}$ is finitely generated, so is the $R$-module $M / r R$. Therefore, $M$ is a finitely generated ideal of $R$, and since $R$ is quasi-local of Krull dimension $1, R$ is a Noetherian domain.

Finally, suppose that $R=A+\mathfrak{m} R$, and let $\phi: \widehat{A} \rightarrow \widehat{R}$ denote the canonical map. We claim that $\widehat{R}=\phi(\widehat{A})+\mathfrak{m} \widehat{R}$. Suppose that $\left\langle r_{i}+\mathfrak{m}^{i} R\right\rangle \in \widehat{R}$. (We are viewing $\widehat{R}$ here as a subring of $\prod_{i} R / \mathfrak{m}^{i} R$.) Then for each $i>0$, there exists $m_{i} \in \mathfrak{m} R$ such that $r_{i}=r_{1}+m_{i}$. Since $R=A+\mathfrak{m} R$, there exist $b \in A$ and $m \in \mathfrak{m} R$ such that $r_{1}=b+m$. Therefore,

$$
\left\langle r_{i}+\mathfrak{m}^{i} R\right\rangle=\left\langle r_{1}+m_{i}+\mathfrak{m}^{i} R\right\rangle=\left\langle b+\left(m+m_{i}\right)+\mathfrak{m}^{i} R\right\rangle \in \phi(\widehat{A})+\mathfrak{m} \widehat{R}
$$

and hence $\widehat{R}=\phi(\widehat{A})+\mathfrak{m} \widehat{R}$. So if we reconsider the elements $\omega_{1}, \ldots, \omega_{n}$ whose images generate $\widehat{R} / \mathfrak{m} \widehat{R}$ as an $\widehat{A}$-module, we see that we may assume that $n=1$ and $\omega_{1}=1$. Thus, appealing again to [17, Theorem 8.4], we conclude that $\widehat{R}$ is generated as an $\widehat{A}$-module by $\omega_{1}=1$. Consequently, $\widehat{R}=\phi(\widehat{A})$, so that $\phi$ is a surjection.

We apply the lemmas in the next theorem, which asserts under certain conditions (to be clarified later in Corollaries 5.5 and 5.11) the existence of bad stable rings tightly dominating a given local Noetherian domain. The theorem also gives a representation of all such stable rings.

TheOrem 5.3. Let $A$ be a local Noetherian domain, not a DVR, that is tightly dominated by a DVR $V$. Let $F$ denote the quotient field of $A$, and let $P$ be the kernel of the canonical homomorphism $\widehat{A} \rightarrow \widehat{V}$. Then $P \cap A=0$, 
and the following statements are equivalent for any ring $R$ properly between $A$ and $V$.

(1) $R$ is a bad stable ring (necessarily Noetherian, by Lemma 5.2) tightly dominating $A$.

(2) $R=F \cap(\widehat{A} / J)$ for some P-primary ideal $J$ of $\widehat{A}$ containing $P^{2}$.

Moreover, every bad stable domain between $A$ and $V$ tightly dominating $A$ contains the bad Noetherian stable domain $R=F \cap\left(\widehat{A} / P^{(2)}\right)$, where $P^{(2)}$ is the second symbolic power of $P$.

Proof. It is clear that $P \cap A=0$, since $V$ is a DVR dominating $A$. First, suppose that $R$ is a bad stable ring properly between $A$ and $V$ that tightly dominates $A$. Then $R$ has maximal ideal $\mathfrak{m} R$, where $\mathfrak{m}$ is the maximal ideal of $A$. Since $R$ tightly dominates $A$, we have by Lemma 5.2 that $R$ is a Noetherian domain and that the canonical mapping $\widehat{A} \rightarrow \widehat{R}$ is a surjection. Let $J$ denote the kernel of this mapping, so that $\widehat{R} \cong \widehat{A} / J$ and $J \subseteq P$. Let $Q$ be the prime ideal of $\widehat{R}$ corresponding to $P / J$. By Lemma 5.2 , the canonical mapping $\widehat{A} \rightarrow \widehat{V}$ is a surjection, so that $\widehat{A} / P \cong \widehat{V}$, and hence $\widehat{A} / P \cong \widehat{R} / Q$ is a DVR. Now since $R$ has Krull dimension 1 , the $\mathfrak{m}$-adic and ideal topologies on $R$ are the same, so $\widehat{R}$ can be viewed as the completion of $R$ in the ideal topology. Thus, by Theorem 3.4, there is a prime ideal $Q^{\prime}$ of $\widehat{R}$ such that $\left(Q^{\prime}\right)^{2}=0$ and $\widehat{R} / Q^{\prime}$ is a DVR. Since then $F \cap \widehat{R} / Q^{\prime}$ is another DVR containing $R$ and the normalization of $R$ is the DVR $V$, this forces $V=F \cap \widehat{R} / Q^{\prime}$. Consequently, by Lemma 5.1, $Q=Q^{\prime}$, and hence $Q^{2}=0$, which implies that $P^{2} \subseteq J \subseteq P$.

To see next that $J$ is $P$-primary, note that since the $\mathfrak{m}$-adic and ideal topologies agree on $R$, then $\widehat{R} \cong \widehat{A} / J$ is a torsion-free $A$-module (see [15, Theorem 2.1, p. 11]). Thus, for all $0 \neq b \in A,\left(J:_{\widehat{A}} b\right)=J$. We claim that the latter property implies that $J$ is $P$-primary. Indeed, since $P^{2} \subseteq J$ and since $\widehat{A} / J$ has Krull dimension 1 , the only prime ideals of $\widehat{A}$ containing $J$ are $P$ and the maximal ideal $\mathfrak{m} \widehat{A}$ of $\widehat{A}$. Thus, to show that $J$ is $P$-primary, it suffices to observe that $\mathfrak{m} \widehat{A}$ is not an associated prime of $J$. For if $x \in \widehat{A}$ with $\mathfrak{m} \widehat{A}=\left(J:_{\widehat{A}} x\right)$, then choosing $0 \neq a \in \mathfrak{m}$, we have $x \in\left(J:_{\widehat{A}} a\right)=J$, contrary to the choice of $x$. Therefore, the only associated prime of $J$ is $P$, and hence $J$ is $P$-primary. Finally, to finish the verification of (2), we need only observe that $R=F \cap(\widehat{A} / J)$. Since $J$ is $P$-primary and $P \cap A=0$, then as discussed at the beginning of this section, $F$ can be identified with a subring of the total quotient ring of $\widehat{A} / J$. Moreover, since $\widehat{R} \cong \widehat{A} / J$, then $\widehat{A} / J$ is faithfully flat over $R$, from which (2) now follows. 
Conversely, suppose that $J$ is a $P$-primary ideal of $\widehat{A}$ such that $P^{2} \subseteq J$ and $R=F \cap(\widehat{A} / J)$. We prove that $R$ is a bad Noetherian stable domain tightly dominating $A$. Since $P \cap A=0$, we may appeal to Lemma 5.1 to obtain that the ring $R=F \cap(\widehat{A} / J)$ is a Noetherian domain of Krull dimension 1 and that the canonical $A$-algebra homomorphism $\widehat{A} \rightarrow \widehat{R}$ is surjective with kernel $I$. Thus, since $\mathfrak{m} \widehat{A}$ is the maximal ideal of $\widehat{A}$, the maximal ideal of $\widehat{R}$ is $\mathfrak{m} \widehat{R}$. Also, since $R$ has Krull dimension 1 and since $\mathfrak{m}$ is a finitely generated ideal of $A$, the $\mathfrak{m}$-adic and ideal topologies agree on $R$, so by Theorem 3.4 , $R$ is a bad stable domain, since $\widehat{A} / P$ is a DVR and $(P / J)^{2}=0$. To see that $\mathfrak{m} R$ is the maximal ideal of $R$, we view $R$ as a subring of $\widehat{R}$. Then since $\mathfrak{m} \widehat{R}$ is the maximal ideal of $\widehat{R}$ and $\widehat{R} / \mathfrak{m} \widehat{R}$ and $R / \mathfrak{m} R$ are isomorphic as rings, it follows that $\mathfrak{m} R$ is the maximal ideal of $R$. In summary, $R=F \cap(\widehat{A} / J)$ is a bad Noetherian stable domain with maximal ideal $\mathfrak{m} R$. Thus, since $V=A+\mathfrak{m} V$ and $\mathfrak{m} R$ is the maximal ideal of $R$, it follows that $R=A+\mathfrak{m} R$, so that $R$ tightly dominates $A$. Thus, (2) implies (1).

Next we observe that the ring $R=F \cap\left(\widehat{A} / P^{(2)}\right)$ is contained in each bad stable ring between $A$ and $V$ that tightly dominates $A$. Let $R^{\prime}$ be such a ring. Then by the equivalence of (1) and (2), there exists a $P$-primary ideal $J$ of $\widehat{A}$ such that $P^{2} \subseteq J$ and $R^{\prime}=F \cap(\widehat{A} / J)$. Since $P^{(2)} \subseteq J$, it follows that $R \subseteq R^{\prime}$

Finally, we show that the embedding dimension of $R=F \cap\left(\widehat{A} / P^{(2)}\right)$ is one more than the embedding dimension of $\widehat{A}_{P}$. Since $P^{(2)}$ is a $P$-primary ideal, it follows that $P / P^{(2)}$ is a torsion-free $\widehat{A} / P$-module. Therefore, $P / P^{(2)}$ is a finitely generated free module over the DVR $\widehat{A} / P$. The rank of this free module is the dimension of the $\left(\widehat{A}_{P} / P_{P}\right)$-vector space $P_{P} / P_{P}^{(2)}=P_{P} / P_{P}^{2}$, and the dimension of this vector space is in turn the embedding dimension, $n$, of $\widehat{A}_{P}$. Thus, the ideal $P / P^{(2)}$ of $\widehat{A} / P^{(2)}$ is minimally generated by $n$ elements. Passing now from $\widehat{A} / P^{(2)}$ to the isomorphic ring $\widehat{R}$ (see Lemma 5.1), there exists a prime $Q$ of $\widehat{R}$ such that $Q^{2}=0, Q$ is minimally generated by $n$ elements, and $\widehat{R} / Q$ is a DVR. Let $t$ be in the maximal ideal $M$ of $\widehat{R}$ such that $M=t \widehat{R}+Q$. We claim that $\left(t \widehat{R}+M^{2}\right) \cap\left(Q+M^{2}\right)=M^{2}$. Let $x \in \widehat{R}$, let $m_{1}, m_{2} \in M^{2}$, and let $q \in Q$ such that $x t+m_{1}=q+m_{2}$. If $x \in M$, then $x t \in M^{2}$, so that $x t+m_{1} \in M^{2}$, as claimed. Otherwise, $x$ is a unit in $\widehat{R}$, and hence $t \in Q+M^{2}$. But then $M=t \widehat{R}+Q \subseteq Q+M^{2}$, which is impossible since $\widehat{R} / Q$ is a DVR with maximal ideal $M / Q$. Therefore, $\left(t \widehat{R}+M^{2}\right) \cap\left(Q+M^{2}\right)=M^{2}$, and hence

$$
M / M^{2}=\left(t \widehat{R}+M^{2}\right) / M^{2} \oplus\left(Q+M^{2}\right) / M^{2} .
$$


Moreover, by Lemma 3.3, there exists $x \in M$ such that $M^{2}=x M$. Therefore, $\left(Q+M^{2}\right) / M^{2} \cong Q /(Q \cap x M)=Q / x Q$. However, $x Q \subseteq M Q \subseteq M^{2} \cap Q=$ $x M \cap Q=x Q$, so $x Q=M Q$, and hence $\left(Q+M^{2}\right) / M^{2} \cong Q / M Q$. Thus, the dimension of the $\widehat{R} / M$-vector space $\left(Q+M^{2}\right) / M^{2}$ is $n$, which using Nakayama's lemma proves that $M$ is minimally generated by $n+1$ elements. Since the embedding dimension of $R$ and $\widehat{R}$ agree, the proof is complete.

REMARK 5.4. Theorem 5.3 does not account for all of the bad stable domains between $A$ and $V$. For example, if we let $R$ be as in (2), then the ring $R_{1}=\operatorname{End}(\mathfrak{m} R)$ is a stable domain between $R$ and $V$ (see Theorem 4.4), but its maximal ideal is not extended from $\mathfrak{m}$, a necessary condition in order for the ring to fall into the classification in the theorem. Indeed, $\mathfrak{m} R_{1}=\mathfrak{m} R$, so that if $\mathfrak{m} R_{1}$ is the maximal ideal of $R_{1}$, then since it is stable, it is necessarily principal in $R_{1}$. But then $R_{1}$ is a DVR, and hence $R_{1}=V$, which forces $V$ to be a fractional ideal of $R$. This is impossible since $V / R$ is a divisible $R$-module. (Divisibility follows from the fact that $V$ is a DVR with $V=$ $R+\mathfrak{m} V$.) Therefore, the ring $R_{1}$ cannot be described as in Theorem 5.3(1). This argument shows that, in general, a bad stable domain that tightly dominates a quasi-local domain $A$ will have stable overrings that do not tightly dominate $A$.

COROLLARY 5.5. The following statements are equivalent for a local Noetherian domain $A$ that is not a DVR.

(1) There is a prime ideal $P$ of $\widehat{A}$ such that $P \cap A=0$ and $\widehat{A} / P$ is a $D V R$.

(2) A is tightly dominated by a DVR.

(3) A is tightly dominated by a bad Noetherian stable domain.

(4) A is tightly dominated by a bad 2-generator domain.

Proof. (1) $\Rightarrow(2)$ Let $V=F \cap(\widehat{A} / P)$, where $F$ denotes the quotient field of $A$. Then since $\widehat{A} / P$ is a DVR with maximal ideal generated by the image of the maximal ideal $\mathfrak{m}$ of $A$, and since the residue field of $\widehat{A} / P$ is $A / \mathfrak{m}$, it follows that $V$ is a DVR with maximal ideal $\mathfrak{m} V$ and residue field $A / \mathfrak{m}$. Therefore, $V$ tightly dominates $A$.

$(2) \Rightarrow(3)$ By Theorem 5.3, $R=F \cap\left(\widehat{A} / P^{(2)}\right)$ is a bad Noetherian stable domain that tightly dominates $A$.

$(3) \Rightarrow(4)$ Let $R$ be a bad Noetherian stable domain that tightly dominates $A$. Apply Theorem 4.4 to the ring $R$ in (3) to obtain a bad stable domain $T$ of embedding dimension 2 such that $T / R$ is a divisible $R$-module. Since the multiplicity and embedding dimension of a local stable ring agree, 
it follows that $T$ is a 2-generator ring. Moreover, the divisibility of $T / R$ implies that $T=R+\mathfrak{m} T$. Thus, since $R=A+\mathfrak{m} R$, we see that $T=A+\mathfrak{m} T$, and hence $T$ tightly dominates $A$.

$(4) \Rightarrow(2)$ Let $R$ be a bad 2-generator domain that tightly dominates $A$. By Proposition 2.1, $\bar{R}$ is a DVR and $\bar{R} / R$ is a divisible $R$-module. Hence, $\bar{R}=R+\mathfrak{m} \bar{R}=A+\mathfrak{m} \bar{R}$, and $\bar{R}$ tightly dominates $A$.

$(2) \Rightarrow(1)$ Suppose that $V$ is a DVR that tightly dominates $A$. Since $V=A+\mathfrak{m} V$, we have by Lemma 5.2 that the canonical mapping $\phi: \widehat{A} \rightarrow \widehat{V}$ is surjective. Since $V$ is a DVR, so is $\widehat{V}$, and hence the kernel $P$ of this mapping is a prime ideal of $\widehat{A}$. If $b \in P \cap A$, then $b \in \mathfrak{m}^{i} V$ for all $i>0$, which since $V$ is a DVR forces $b=0$. Therefore, $P \cap A=0$.

From Theorem 5.3 we deduce a bound on the embedding dimension of bad stable rings dominating an excellent local Noetherian domain, as follows.

Corollary 5.6. Let $A$ be an excellent local Noetherian domain. If $A$ has dimension $d>1$ and the generic formal fiber of $A$ has dimension $d-1$, then there exists a bad Noetherian stable ring finitely dominating $A$ and having embedding dimension $d$, and every bad stable ring finitely dominating $A$ has embedding dimension at most $d$.

Proof. Since the generic formal fiber has dimension $d-1$, there exists by a theorem of Heinzer, Rotthaus, and Sally [8, Corollary 1.24] a DVR $V$ finitely dominating $A$ (see Corollary 5.11). Since $V$ finitely dominates $A$, there exist $x_{1}, \ldots, x_{n} \in V$ such that $V$ tightly dominates $B:=A\left[x_{1}, \ldots, x_{n}\right]_{\mathbf{n}}$, where $\mathfrak{n}$ is the contraction of the maximal ideal of $V$ to $A\left[x_{1}, \ldots, x_{n}\right]$. Since the residue field of $V$ is finite over that of $A$, the residue field of $B$ is finite also over the residue field of $A$. Thus, since $A$ is universally catenary, the dimension formula implies that the dimension of $B$ is $d$ (see [17, Theorem 15.6, p. 119]). Also, since $A$ is excellent and $B$ is a localization of a finitely generated $A$-algebra, the generic formal fiber of $B$ is regular (see [16, Theorem 77, p. 254]). Therefore, for the prime ideal $P$ of $\widehat{B}$ corresponding to $V$ as in Theorem 5.3, the ring $\widehat{B}_{P}$ is a regular local ring and hence has embedding dimension $d-1$. Applying Theorem 5.3, there exists a bad Noetherian stable ring finitely dominating $A$ and having embedding dimension $d$. Now let $S$ be a bad stable ring finitely dominating $A$. Then by Proposition $2.1, S$ is tightly dominated by a DVR $U$, and as above, $S$ tightly dominates a local $d$ dimensional Noetherian domain $B$ essentially of finite type over $A$. It follows that $U$ tightly dominates $B$, and by Theorem 5.3 and the above argument, 
there exists a bad stable ring of embedding dimension $d$ contained in $S$. Hence, by Theorem 4.4, $S$ has embedding dimension at most $d$.

Corollary 5.7. Let $k$ be a field, and let $A$ be an affine $k$-domain with Krull dimension $d>1$ and quotient field $F$. Then there exists a bad stable ring between $A$ and $F$ having embedding dimension $d$ and residue field finite over $k$. Moreover, every bad stable ring between $A$ and $F$ having residue field finite over $k$ has embedding dimension at most $d$.

Proof. Matsumura [18, Theorem 1] has shown that the generic formal fiber of a $d$-dimensional domain essentially of finite type over a field has dimension $d-1$. Thus, by Corollary 5.6, there exists a bad stable ring between $A$ and $F$ having embedding dimension $d$ and residue field finite over $k$. If $R$ is a bad stable ring between $A$ and $F$ having residue field finite over $k$, then $R$ finitely dominates $A_{M \cap A}$, where $M$ is the maximal ideal of $R$. Since $A_{M \cap A}$ is excellent of dimension $d$, Corollary 5.6 implies that $R$ has embedding dimension at most $d$.

Theorem 5.3 shows that there exists a smallest bad stable domain $R$ between $A$ and $V$, as long as one restricts to those domains $R$ tightly dominating $A$. We remove this restriction of tight domination by $R$ and show in Theorem 5.9 that among all bad stable rings between $A$ and $V, R=\operatorname{Ker} d_{V / A}$ is the smallest. (It follows easily from properties of derivations that their kernels are rings.)

Lemma 5.8. Let $A$ be a quasi-local domain tightly dominated by a DVR $V$, and let $R=\operatorname{Ker} d_{V / A}$. Then $V / R$ and $\Omega_{V / A}$ are isomorphic as $R$-modules, and if $R \subsetneq V$, then $R$ is a bad stable ring contained in every bad stable ring between $A$ and $V$.

Proof. Let $\mathfrak{m}$ denote the maximal ideal of $A$. Since $V$ tightly dominates $A$, we have $V=A+\mathfrak{m}^{i} V$ for all $i>0$. This implies that $V / A$ is a divisible $A$ module. For if $0 \neq a \in \mathfrak{m}$, then since $a V$ and $\mathfrak{m} V$ are primary with respect to the maximal ideal of $V$, there exists $i>0$ such that $\mathfrak{m}^{i} V \subseteq a V$, and hence $V=$ $A+a V$, which implies that $V / A$ is a divisible $A$-module. Next, an argument such as that in the proof of Lemma $3.2(4) \Rightarrow(5)$ shows then that $d_{V / A}(V)=$ $\Omega_{V / A}$, and hence, since $d_{V / A}$ is an $R$-module homomorphism (indeed, since $R=\operatorname{Ker} d_{V / A}$, then $d_{V / A}$ is $R$-linear), then $V / R \cong \Omega_{V / A}$ as $R$-modules. To complete the proof, we assume that $R \neq V$. First we claim that $R$ is a bad stable domain. As a homomorphic image of $V / A, V / R$ is a torsion divisible $A$-module. But since $\Omega_{V / A}$ is a $V$-module and $V / R \cong \Omega_{V / A}$ as $R$-modules, 
it follows that the $R$-module $V / R$ admits a $V$-module structure. Also, since $V / A$ is a divisible $A$-module and $A$ and $R$ share the same quotient field, then $V / R$ is a divisible $R$-module. Therefore, by Lemma $3.2, R \subseteq V$ is a quadratic extension. Since $R \neq V$, we have by Proposition 2.1 that $R$ is a bad stable ring. Applying Proposition 2.1 again shows that every ring $R^{\prime}$ with $R \subseteq R^{\prime} \subsetneq V$ is a bad stable ring.

Now suppose that $R^{\prime}$ is a bad stable ring with $A \subseteq R^{\prime} \subseteq V$. Then by Proposition 2.1, the normalization of $R^{\prime}$ is a DVR, so necessarily $V$ is the integral closure of $R^{\prime}$. Also, by Proposition 2.1, $R^{\prime} \subseteq V$ is a quadratic extension, so since $V / R^{\prime}$ is a divisible $R^{\prime}$-module, Lemma 3.2(5) implies that the exterior differential $d_{V / R^{\prime}}$ maps onto $\Omega_{V / R^{\prime}}$ with kernel $R^{\prime}$. However, since $d_{V / R^{\prime}}$ is an $A$-linear derivation, there exists, as discussed in Section 1 , a $V$-module homomorphism $\alpha: \Omega_{V / A} \rightarrow \Omega_{V / R^{\prime}}$ such that $d_{V / R^{\prime}}=\alpha \circ d_{V / A}$. Thus, $R=\operatorname{Ker} d_{V / A} \subseteq \operatorname{Ker} d_{V / R^{\prime}}=R^{\prime}$, which completes the proof.

TheOrem 5.9. Let $A$ be a local Noetherian domain, not a DVR, that is tightly dominated by a DVR $V$. Then the ring $R=\operatorname{Ker} d_{V / A}$ is a bad stable domain contained in every bad stable ring between $A$ and $V$.

Proof. In light of Lemma 5.8, all that needs to be shown is that $R$ is a proper subring of $V$. In fact, since $R$ is a subring of every bad stable domain between $A$ and $V$, we need only show that there exists a bad stable domain between $A$ and $V$. The existence of such a ring is guaranteed by Theorem 5.3, so the proof is complete.

We use the preceding ideas next to characterize the local Noetherian domains which are finitely dominated by a bad stable ring. Matlis [15, Theorem 14.16] has proved that if $A$ is an analytically ramified local Noetherian domain of Krull dimension 1, then there exists a bad 2-generator ring $R$ between $A$ and its quotient field. In Theorem 5.10 we recover a version of Matlis's theorem from a different point of view.

THEOREM 5.10 ([15, Theorem 14.16]). Every 1-dimensional analytically ramified local Noetherian domain is finitely dominated by a bad 2-generator ring.

Proof. We first show that there exists an analytically ramified local Noetherian ring finitely dominating $A$ whose normalization is a DVR. Since $\bar{A}$ is a Dedekind domain, it has only finitely many maximal ideals. For each of these maximal ideals, choose an element in it but in no other maximal ideal, and let $T$ be the $A$-algebra generated by these finitely many elements. 
Then $T$ has the same number of maximal ideals as $\bar{A}$. Since $\bar{A}$ is not a finite $T$-module (if it were, it would force $\bar{A}$ to be a finite $A$-module, contrary to assumption), there exists a maximal ideal $M$ of $T$ such that the $\operatorname{ring} T_{M}$ is analytically ramified. Indeed, suppose otherwise. Then each localization of $T$ at a maximal ideal $M$ is analytically unramified, so that each ring $\bar{A}_{M}$ is a fractional ideal of $T_{M}$. But this then implies that there exists an element $t$ of $T$ such that $t \bar{A} \subseteq T$, a contradiction to the fact that $\bar{A}$ is not a finite module over the Noetherian ring $T$. Thus, there is a maximal ideal $M$ of $T$ such that the ring $A^{\prime}:=T_{M}$ is an analytically ramified local Noetherian domain with integral closure the DVR $V:=\bar{A}_{N}$, where $N$ is the unique maximal ideal of $\bar{A}$ lying over $M$. Finally, to see that $A^{\prime}$ finitely dominates $A$, let $\mathfrak{m}^{\prime}$ be the maximal ideal of $A^{\prime}$. Since $A^{\prime}=T_{M}$, we have $A^{\prime}=T+\mathfrak{m}^{\prime}$, and since $A^{\prime}$ has Krull dimension 1 and its maximal ideal $\mathfrak{m}^{\prime}$ is finitely generated, there exists $k>0$ such that $\left(\mathfrak{m}^{\prime}\right)^{k} \subseteq \mathfrak{m} A^{\prime}$. Now since $A^{\prime}=T+\mathfrak{m}^{\prime}$ and $T$ is a finite $A$-module, then $A^{\prime} / \mathfrak{m}^{\prime}$ is a finite $A$-module, and hence it follows that $A^{\prime} /\left(\mathfrak{m}^{\prime}\right)^{k}$ is a finite $A$-module. Therefore, since $A^{\prime} / \mathfrak{m} A^{\prime}$ is an $A$-homomorphic image of $A^{\prime} /\left(\mathfrak{m}^{\prime}\right)^{k}$, we conclude that $A^{\prime} / \mathfrak{m} A^{\prime}$ is a finite $A$-module, and this proves that $A^{\prime}$ is an analytically ramified local Noetherian ring that finitely dominates $A$ and the normalization of $A^{\prime}$ is the DVR $V$.

We note next that there exists a finite extension $A^{\prime \prime}$ of $A^{\prime}$ such that $V$ tightly dominates $A^{\prime \prime}$. Since $V$ finitely dominates $A^{\prime}$, there exist $x_{1}, \ldots, x_{n} \in$ $V$ such that $V=A^{\prime} x_{1}+\cdots+A^{\prime} x_{n}+\mathfrak{m} V$. Since $V$ is the normalization of $A^{\prime}$, the ring $A^{\prime \prime}:=A^{\prime}\left[x_{1}, \ldots, x_{n}\right]$ is a finite extension of $A^{\prime}$ having normalization $V$, and since $V$ is not a finite $A^{\prime}$-module, it follows that $V$ is not a finite $A^{\prime \prime}$-module. Thus, $A^{\prime \prime}$ is a 1-dimensional analytically ramified local Noetherian domain that is tightly dominated by $V$. We now apply either Theorem 5.3 or Theorem 5.9 to obtain a bad stable ring $R$ with $A^{\prime \prime} \subseteq R \subseteq V$. Applying Theorem 4.4, we may in fact assume that $R$ has embedding dimension 2 , and hence since in a stable ring multiplicity and embedding dimension agree, $R$ is a bad 2-generator ring. Moreover, $R$ and $V$ have the same residue field. Indeed, since $V$ tightly dominates $A^{\prime \prime}, V=A^{\prime \prime}+\mathfrak{m}^{\prime \prime} V$, where $\mathfrak{m}^{\prime \prime}$ is the maximal ideal of the 1-dimensional local domain $A^{\prime \prime}$. Thus, since the maximal ideal $M$ of $R$ contains $\mathfrak{m}^{\prime \prime}$, it follows that $V=R+M V$, and hence $V / M V \cong R / M$. Therefore, $R$ and $V$ have the same residue field. Similarly, since $V=A^{\prime \prime}+\mathfrak{m}^{\prime \prime} V$, then $V$ and $A^{\prime \prime}$ have the same residue field, and therefore, $A^{\prime \prime}$ and $R$ have the same residue field. Now $R / M$ is a cyclic $A^{\prime \prime}$-module, so since $A^{\prime \prime}$ is a finite $A^{\prime}$-module, $R / M$ is a finite $A^{\prime} / \mathfrak{m} A^{\prime}$-module. Thus, since $A^{\prime} / \mathfrak{m} A^{\prime}$ is a finite $A$-module, we see that $R / M$ is a finite $A$-module. 
Moreover, since $M$ is a finitely generated ideal of $R$ (necessarily $R$ is Noetherian, since it is an overring of the 1-dimensional Noetherian domain $A$ ), there exists $k>0$ such that $M^{k} \subseteq \mathfrak{m} R$. It follows that $R / M^{k}$ is a finite $A$-module, so since $R / \mathfrak{m} R$ is an $A$-homomorphic image of $R / M^{k}$, we conclude that $R / \mathfrak{m} R$ is a finite $A$-module.

Thus, a 1-dimensional local Noetherian domain $A$ is finitely dominated by a bad stable ring if and only if $A$ is analytically ramified. In higher dimensions, we have the following.

Corollary 5.11. Let $A$ be a local Noetherian domain with dimension $d>1$. Then the following statements are equivalent.

(1) A is finitely dominated by a bad Noetherian stable ring.

(2) A is finitely dominated by an analytically ramified 1-dimensional local Noetherian ring.

(3) A is tightly dominated by an analytically ramified 1-dimensional local Noetherian ring.

(4) A is finitely dominated by a DVR.

(5) The dimension of the generic formal fiber of $A$ is $d-1$.

If also $A$ is excellent, then the stable ring in (1) can be chosen to have embedding dimension d but no bigger. Moreover, these five equivalent conditions are satisfied when $A$ is essentially of finite type over a field and has dimension $d>1$.

Proof. (1) $\Rightarrow(2)$ This is clear.

$(2) \Rightarrow(4)$ If $R$ is a 1-dimensional analytically ramified local Noetherian ring that finitely dominates $A$, then by Theorem 5.10, $R$ is finitely dominated by a bad stable ring $S$. The $\operatorname{ring} S$ in turn is tightly dominated by a DVR $V$ (see Proposition 2.1), and hence $V$ finitely dominates $A$.

$(4) \Rightarrow(5)$ This is due to Heinzer, Rotthaus, and Sally [8, Corollary 2.4].

$(5) \Rightarrow(3)$ By $(5)$, there exists a height $d-1$ prime ideal $P$ of $\widehat{A}$ such that $\widehat{A} / P$ has Krull dimension 1 and $A \cap P=0$. Since $d>1, P$ is not a minimal prime ideal of $\widehat{A}$. With the aim of applying Lemma 5.1, observe that $P^{(2)} \subsetneq P$, since otherwise $P^{2} \widehat{A}_{P}=P \widehat{A}_{P}$, which, since $P$ is finitely generated, implies that $P \widehat{A}_{P}=0$ and hence that $P$ is a minimal prime ideal of $\widehat{A}$, a contradiction. Therefore, we may apply Lemma 5.1 to obtain that $R:=\left(\widehat{A} / P^{(2)}\right) \cap F$ is a Noetherian domain of Krull dimension 1 with $\widehat{R} \cong \widehat{A} / P^{(2)}$. Also, $R$ is analytically ramified because $P \neq P^{(2)}$, so that $\widehat{R}$ contains nilpotents. Since $\widehat{A} / P^{(2)}$ has a unique minimal prime ideal, namely, 
$P / P^{(2)}$, it follows that the normalization of $R$ is a DVR (see [15, Theorem 10.5]). Thus, we have constructed a 1-dimensional analytically ramified local Noetherian domain $R$ that birationally dominates $A$. Moreover, since $\widehat{R} \cong \widehat{A} / P^{(2)}$, it follows that $\widehat{R}$ and $\widehat{A}$, and hence $R$ and $A$, have the same residue field and that $\mathfrak{m} \widehat{R}$ is the maximal ideal of $\widehat{R}$. Consequently, $\mathfrak{m} R$ is the maximal ideal of $R$, and hence since $R$ and $A$ have the same residue field, $R=A+\mathfrak{m} R$. Therefore, $R$ tightly dominates $A$.

$(3) \Rightarrow(1)$ Let $R$ be an analytically ramified 1-dimensional local Noetherian ring that finitely dominates $A$. Then by Theorem $5.10, R$ is finitely dominated by a bad Noetherian stable ring, and hence this ring finitely dominates $A$.

If also $A$ is excellent and (1)-(5) hold, then by Corollary 5.6, $A$ is finitely dominated by a bad stable ring of embedding dimension $d$ and every bad stable ring finitely dominating $A$ has embedding dimension at most $d$. Finally, the last assertion of the corollary, that all five conditions are equivalent when $A$ is essentially of finite type over a field, follows from [18, Theorem 2], where it is shown that for such a ring the dimension of the generic formal fiber is $d-1$.

REMARK 5.12. Statements (4) and (5) are equivalent also when $A$ has Krull dimension 1 (see [8, Corollary 2.4]).

Acknowledgment. I would like to thank the referees for helpful comments that improved the presentation of this article.

\section{REFERENCES}

[1] Y. Akizuki, Einige Bemerkungen über primäre Integritätsbereiche mit Teilerkettensatz, Proc. Phys. Math. Soc. Japan 17 (1935), 327-336.

[2] H. Bass, On the ubiquity of Gorenstein rings, Math Z. 82 (1963), 8-28. MR 0153708.

[3] J. A. Drozd and V. V. Kiričenko, The quasi-Bass orders (in Russian), Izv. Akad. Nauk SSSR Ser. Mat. 6 (1972), 328-370; English translation in Math. USSR-Izv. 6 (1972), 323-365. MR 0304411.

[4] S. El Baghdadi and S. Gabelli, Ring-theoretic properties of PvMDs, Comm. Algebra 35 (2007), 1607-1625. MR 2317633. DOI 10.1080/00927870601169283.

[5] L. Fuchs and L. Salce, Modules over non-Noetherian Domains, Math. Surveys Monogr. 84, Amer. Math. Soc., Providence, 2001. MR 1794715.

[6] S. Gabelli and G. Picozza, Star stable domains, J. Pure Appl. Algebra 208 (2007), 853-866. MR 2283430. DOI 10.1016/j.jpaa.2006.03.020.

[7] W. Heinzer, D. Lantz, and K. Shah, The Ratliff-Rush ideals in a Noetherian ring, Comm. Algebra 20 (1992), 591-622. MR 1146317. DOI 10.1080/00927879208824359.

[8] W. Heinzer, C. Rotthaus, and J. D. Sally, Formal fibers and birational extensions, Nagoya Math. J. 131 (1993), 1-38. MR 1238631. 
[9] S. Kabbaj and A. Mimouni, t-Class semigroups of integral domains, J. Reine Angew. Math. 612 (2007), 213-229. MR 2364057. DOI 10.1515/CRELLE.2007.088.

[10] W. Krull, Dimensionstheorie in Stellenringen, J. Reine Angew. Math. 179 (1938), 204-226.

[11] J. Lipman, Stable ideals and Arf rings, Amer. J. Math. 93 (1971), 649-685. MR 0282969.

[12] E. Matlis, Injective modules over Noetherian rings, Pacific J. Math. 8 (1958), 511528. MR 0099360.

[13] - Injective modules over Prüfer rings, Nagoya Math. J. 15 (1959), 57-69. MR 0109840.

[14] — Torsion-Free Modules, University of Chicago Press, Chicago, 1972. MR 0344237.

[15] — 1-Dimensional Cohen-Macaulay Rings, Lecture Notes in Math. 327, Springer, Berlin, 1973. MR 0357391.

[16] H. Matsumura, Commutative Algebra, 2nd ed., Math. Lecture Note Series 56, Benjamin/Cummings, Reading, Mass., 1980. MR 0575344.

[17] - Commutative Ring Theory, Cambridge Stud. Adv. Math. 8, Cambridge University Press, Cambridge, 1986. MR 0879273.

[18] - "On the dimension of formal fibres of a local ring" in Algebraic Geometry and Commutative Algebra, Vol. I, Kinokuniya, Tokyo, 1988, 261-266. MR 0977763.

[19] A. Mimouni, Ratliff-Rush closure of ideals in integral domains, Glasg. Math. J. 51 (2009), 681-689. MR 2534017. DOI 10.1017/S0017089509990097.

[20] R. Nagasawa, Some remarks on one-dimensional local domains, Publ. Res. Inst. Math. Sci. 11 (1975/76), 21-30. MR 0441955.

[21] M. Nagata, Local Rings, Interscience Tracts Pure Appl. Math. 13, Wiley, New York, 1962. MR 0155856.

[22] B. Olberding, On the classification of stable domains, J. Algebra 243 (2001), 177197. MR 1851660. DOI 10.1006/jabr.2001.8832.

[23] - Stability, duality, 2-generated ideals and a canonical decomposition of modules, Rend. Semin. Mat. Univ. Padova 106 (2001), 261-290. MR 1876223.

[24] - "Stability of ideals and its applications" in Ideal Theoretic Methods in Commutative Algebra (Columbia, Mo., 1999), Lecture Notes Pure Appl. Math. 220, Dekker, New York, 2001, 319-341. MR 1836608.

[25] - On the structure of stable domains, Comm. Algebra 30 (2002), 877-895. MR 1883031. DOI 10.1081/AGB-120013188.

[26] —, A counterpart to Nagata idealization, J. Algebra 365 (2012), 199-221. MR 2928459. DOI 10.1016/j.jalgebra.2012.05.002.

[27] - One-dimensional bad Noetherian rings, to appear in Trans. Amer. Math. Soc., preprint, arXiv:1208.2913 [math.AC]

[28] G. Picozza and F. Tartarone, Flat ideals and stability in integral domains, J. Algebra 324 (2010), 1790-1802. MR 2678822. DOI 10.1016/j.jalgebra.2010.07.021.

[29] J. D. Sally and W. V. Vasconcelos, Stable rings and a problem of Bass, Bull. Amer. Math. Soc. (N.S.) 79 (1973), 574-576. MR 0311643.

[30] - Stable rings, J. Pure Appl. Algebra 4 (1974), 319-336. MR 0409430.

[31] F. K. Schmidt, Über die Erhaltung der Kettensätze der Idealtheorie bei beliebigen endlichen Körpererweiterungen, Math. Z. 41 (1936), 443-450. MR 1545632. DOI 10. 1007/BF01180433. 
[32] L. Sega, Ideal class semigroups of overrings, J. Algebra 311 (2007), 702-713. MR 2314730. DOI 10.1016/j.jalgebra.2006.10.030.

[33] P. Zanardo, The class semigroup of local one-dimensional domains, J. Pure Appl. Algebra 212 (2008), 2259-2270. MR 2426506. DOI 10.1016/j.jpaa.2008.03.015.

[34] - Algebraic entropy of endomorphisms over local one-dimensional domains, J. Algebra Appl. 8 (2009), 759-777. MR 2597279. DOI 10.1142/S0219498809003618.

Department of Mathematical Sciences

New Mexico State University

Las Cruces, New Mexico 88003-8001

USA

olberdin@nmsu.edu 